\title{
Collective Magnetic Behavior of Graphene Nanohole Superlattices
}

\author{
Decai Yu, Elizabeth M. Lupton, Miao Liu, Wei Liu, and Feng Liu ( $\varangle)$ \\ Department of Materials Science and Engineering, University of Utah, Salt Lake City, UT 84112, USA \\ Received: 29 April 2008/ Revised: 19 May 2008/Accepted: 20 May 2008 \\ OTsinghua Press and Springer-Verlag 2008
}

\begin{abstract}
We predict a new class of 2-D crystalline "bulk" magnets-the graphene nanohole (GNH) superlattices with each GNH acting like a "super" magnetic atom, using first principles calculations. We show that such superlattices can exhibit long-range magnetic order above room temperature, with a collective magnetic behavior governed by inter-NH spin-spin interactions in additional to intra-NH spin ordering. Furthermore, magnetic semiconductors can be made by doping magnetic NHs into semiconducting NH superlattices. The possibility of engineering magnetic GNHs for storage media and spintronics applications is discussed.
\end{abstract}

\section{KEYWORDS}

Graphene, magnetism, spin, magnetic semiconductor, superlattice

Nanostructured magnetic materials have a wide range of applications, such as being used for storage media. Magnetism is commonly associated with elements containing localized $\mathrm{d}$ or $\mathrm{f}$ electrons, i.e., the itinerant ferromagnetism [1,2]. In contrast, the elements containing diffuse sp electrons are intrinsically nonmagnetic, but magnetism can be induced in sp-element materials extrinsically by defects and impurities. There have been continuing efforts in searching for new magnetic nanomaterials, and much recent interest has been devoted to magnetism of carbon-based [3-7], especially graphene-based structures [8-18] such as graphene nanoribbons $[8,9,11,18]$ and nanoflakes [16,17]. Graphene nanoribbons $[8,9,11,18]$ and nanoflakes $[16,17]$ with zigzag edges have been shown to exhibit magnetism, originated from the localized edge states that give rise to a high density of states at the Fermi level rendering a spin-polarization instability [1]. However, these structures exhibit only local magnetization, lacking the long-range magnetic order with a well-defined transition temperature. Also, it is practically difficult to control the magnetic properties of individual nanoribbons and nanoflakes and integrate them into devices.

Here we predict a new class of graphene-based magnetic nanostructures, the superlattices of graphene nanoholes (GNHs), which exhibit long-range magnetic order and collective "bulk" magnetism. This allows us to go beyond the current scope limited to the spins within a single nanoribbon or nanoflake. In fact, the superlattices consisting of a periodic array of $\mathrm{NH}$ spins form a unique family of magnetic 2-D crystals with the NH acting like a "super" magnetic atom. Their collective magnetic behavior depends on not only the local intra-NH spin property but also the long-range inter-NH spin-spin interactions. The type of magnetic order, i.e., ferromagnetic (FM) vs antiferromagnetic (AF), can be controlled by using different $\mathrm{NH}$ shapes and superlattice symmetries, and the ordering

Address correspondence to fliu@eng.utah.edu 
temperature can be tuned by $\mathrm{NH}$ size and density well above room temperature. It is also possible to combine magnetic NHs with nonmagnetic semiconducting $\mathrm{NHs}$ to form magnetic semiconductors. Our findings represent a unique organic material exhibiting collective "bulk" magnetism, with significant implications in studying spin-spin interactions in lowdimensional nanostructured systems and in potential applications of magnetic and spintronic materials.

We have performed extensive first-principle calculations to investigate the magnetic properties of GNH superlattices as a function of $\mathrm{NH}$ size and shape as well as of superlattice cell dimensions (i.e., NH density) and symmetries. Our calculations were performed using the pseudopotential planewave method within the spin-polarized generalized gradient approximation as implemented in the VASP code [19]. We used a rhombus supercell in the graphene plane with the cell sizes ranging from $14 \AA x$ $14 \AA$ to $41 \AA \times 41 \AA$ and $a$ vacuum layer of $\sim 10 \AA$. We used a $2 \times 2 \times 1 k$-point mesh for Brillouin zone sampling and a plane wave cutoff of $22.1 \mathrm{Rd}$. The systems contain up to a maximum of 530 atoms. All the carbon atoms on the edge with dangling bonds are terminated by hydrogen atoms. The smallest $\mathrm{H}-\mathrm{H}$ separation on the neighboring $\mathrm{C}$ atoms is found at the corner of NHs to be $1.23 \AA$, much larger than the $\mathrm{H}_{2}$ bond length of $0.74 \AA$. The system is relaxed until the force on each atom is minimized to less than $0.01 \mathrm{eV} / \AA$.

Considering first a single zigzag $\mathrm{NH}$ by examining the intra-NH spin-spin interaction, we found that individual $\mathrm{NH}$ can be viewed as an "inverse structure" of nanoflake or nanoribbon, like an anti-flake or anti-ribbon, with similar spin behavior. We determine the ground-state magnetism of three typical NH shapes-triangular (Fig. 1(a)), rhombus (Fig. 1(b)), and hexagonal (Fig. 1(c)), by comparing the relative stability of ferromagnetic (FM), antiferromagnetic (AF), and paramagnetic (PM) configuration as a function of $\mathrm{NH}$ size. Our calculations show that the ground state is FM for triangular NHs, but AF for rhombus and hexagonal NHs, and their spin densities are shown in Figs. 1(a), (b) and (c), respectively. The magnetic moments are highly concentrated on the edges and decay quickly away from the edge (Fig. 1(d)). Similar decaying behavior has been seen in nanoribbons $[9,11]$ and nanoflakes $[16,17]$. The edge moment increases with increasing NH size (Fig. 1(d), inset).

The triangular NHs have a metastable ferrimagnetic state with two edges having one spin and the other edge

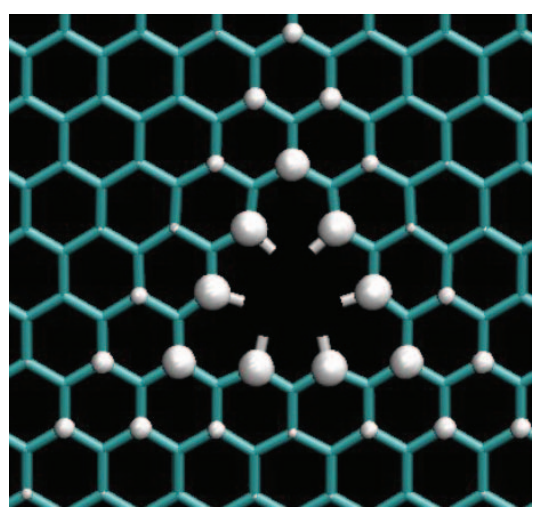

(a)

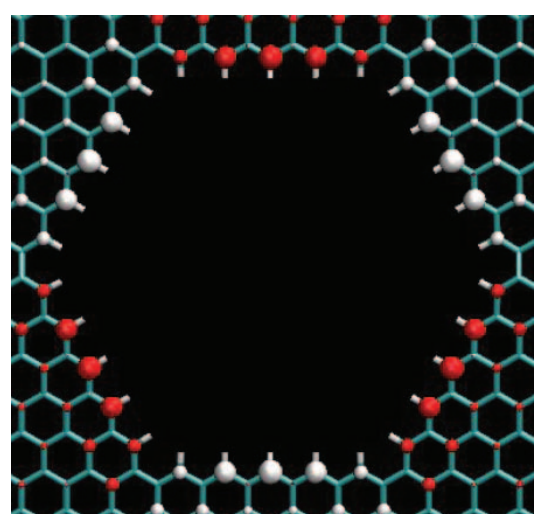

(c)

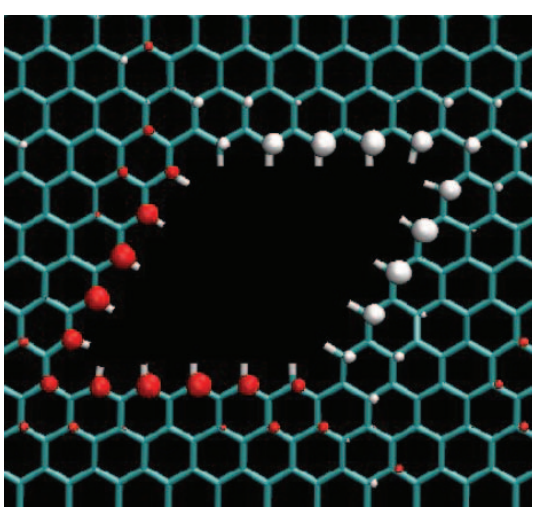

(b)

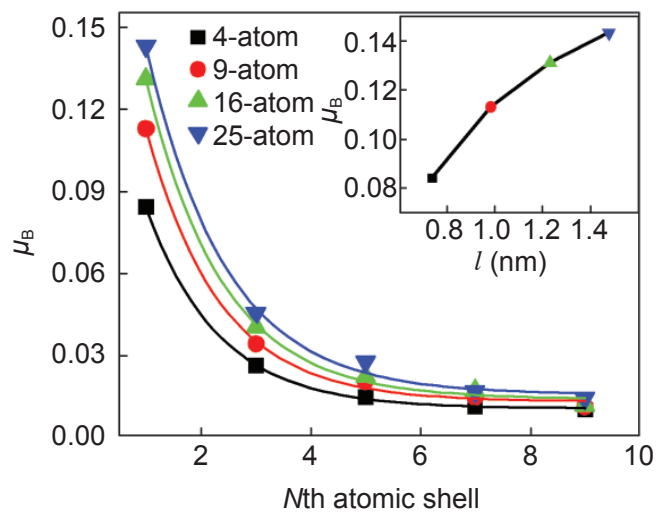

(d)
Figure 1 The ground-state magnetic configurations of different shapes of $\mathrm{NHs}$ : (a) FM triangular $\mathrm{NH}$; (b) AF rhombus $\mathrm{NH}$; (c) AF hexagonal NH. In (a)-(c), white and red balls indicate the up- and downspin density isosurface at $0.02 \mathrm{e} / \AA^{3}$, respectively; blue and white sticks represent $\mathrm{C}-\mathrm{C}$ and $\mathrm{C}-\mathrm{H}$ bonds, respectively. (d) The average local magnetic moment $\left(\mu_{\mathrm{B}}\right)$ per atom in the triangular $\mathrm{NH}$ as a function of distance moving away from the center of $\mathrm{NH}$, measured in atomic shells with the edge atoms as the first shell. The inset shows $\mu_{\mathrm{B}}$ on the edge vs NH size $(l)$ 
having the opposite spin (see online supplementary Fig. S-1). For a 4-atom NH (with four atoms removed from the original lattice), the ferrimagnetic state is $52 \mathrm{meV}$ lower in energy than the FM state, and the latter is $13 \mathrm{meV}$ lower than the PM state. For a 32-atom rhombus $\mathrm{NH}$, the AF state is $89.2 \mathrm{meV}$ lower than the PM state; for a 54-atom hexagonal NH, it is $164.4 \mathrm{meV}$. The energy difference increases with increasing NH size. The triangular NHs favor FM at all sizes, whereas rhombus and hexagonal NHs only become AF when their size is larger than 32 and 96 atoms, respectively. (For smaller sizes, rhombus and hexagonal NHs will relax to the PM state if an AF state was set up initially.) So, the triangular NHs have a stronger tendency toward magnetization.

The magnetic ordering within a single $\mathrm{NH}$ is consistent with both the theorem of itinerant magnetism in a bipartite lattice [20] and the topological frustration model of the $\pi$-bonds [17] counting the unpaired spins in the nonbonding states $[2,17]$. For a system like graphene consisting of two atomic sublattices, each sublattice assumes one spin and the total spin $S$ of the ground state equals $\frac{1}{2}\left|N_{\mathrm{B}}-N_{\mathrm{A}}\right|$ where $N_{\mathrm{B}}\left(N_{\mathrm{A}}\right)$ is the number of atoms B (A) sublattice. Because of the underlying honeycomb lattice symmetry, atoms on the same zigzag edge belong to the same sublattice; while atoms on two different zigzag edges belong to the same sublattice if the two edges are at an angle of $0^{\circ}$ or $120^{\circ}$, but different sublattices if at an angle of $60^{\circ}$ or $180^{\circ}$, as illustrated in online supplementary Fig. S-2. Note that the angle between two edges is defined formally as the angle between the two normal vectors of the edges. Consequently, the triangular $\mathrm{NHs}$ are $\mathrm{FM}$, because all three edges are at $120^{\circ}$ with each other on the same sublattice; the rhombus and hexagonal $\mathrm{NHs}$ are $\mathrm{AF}$, because one-half the edges are on the A-sublattice and another half on the B-sublattice having a $60^{\circ}$ angle between the A- and B-edges. This same argument can be applied to nanoribbons [9] and nanoflakes [16,17].

Next, we consider GNH superlattices (a periodic array of NHs) by examining the inter-NH spin-spin interaction. In principle, one can generate four out of five possible 2-D Bravais lattices of NHs, as shown in Fig. 2. Here, we focus on honeycomb superlattices of triangular NHs (Figs. 3(a) and 3(b)), in which each $\mathrm{NH}$ possesses a net moment acting effectively as one super magnetic atom. The superlattice contains two sublattices of NHs, superimposed on the background of graphene containing two sublattices of atoms. We realize that the NHs on the same sublattice will be FM-coupled because their corresponding edges are at $0^{\circ}$ to each other so that their edge atoms are on the same atomic sublattice. On the other hand, the NHs on different sublattices will be FM-coupled if they are in a parallel configuration (Fig. 3(a)) but AF-coupled if they are in an antiparallel configuration (Fig. 3(b)) when their corresponding edges are at $180^{\circ}$ to each other so that their edge atoms are on different atomic sublattices. These have indeed been confirmed by our

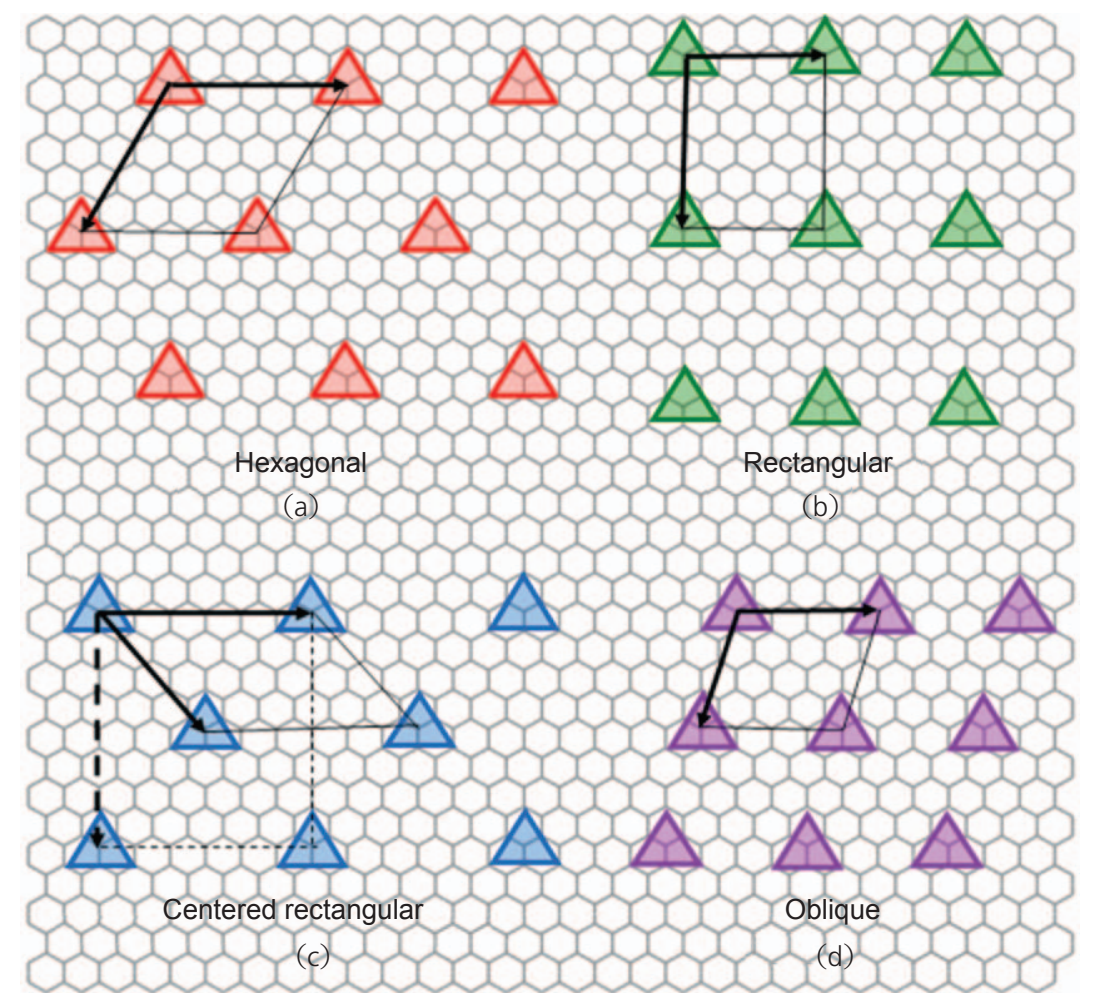

Figure 2 Schematic illustration of four possible types of Bravais lattice of GNHs that can be patterned in graphene with solid arrows and lines marking the primitive cells. (a) hexagonal lattice; (b) rectangular lattice; (c) centered rectangular lattice, the dashed lines mark the conventional cell; (d) oblique lattice. Note that the square lattice is not possible 
first-principle calculations. Independent of $\mathrm{NH}$ size and supercell dimension, the FM state is favored for parallel configurations but the AF state is favored for antiparallel configurations. In both cases, the spinpolarization splits the edge states opening a gap at the Fermi energy $[9,17]$ (see supplementary Fig. S-3). The total spin $S$ in one unit cell equals to $\frac{1}{2}\left|N_{\mathrm{B}}-N_{\mathrm{A}}\right|$; it increases linearly in the FM parallel configuration but remains zero in the AF antiparallel configuration with increasing $\mathrm{NH}$ size.

The collective magnetic behavior of a GNH superlattice depends on inter-NH spin-spin interaction. There exists super exchange interaction between the NH spins mediated by the otherwise "nonmagnetic" graphene lattice, in addition to the spin coupling defined by the underlying bipartite lattice. In Fig. 3(c), we plot $\Delta E_{\mathrm{pc}}=E(\mathrm{FM})-E(\mathrm{PM})$ for the FM parallel configuration and $\Delta E_{\mathrm{ac}}=E(\mathrm{AF})-$
$E(\mathrm{PM})$ for the AF antiparallel configuration as a function of cell dimension $(L)$, i.e., the $\mathrm{NH}-\mathrm{NH}$ separation. $\left|\Delta E_{\mathrm{pc}}\right|$ increases while $\left|\Delta E_{\mathrm{ac}}\right|$ decreases with decreasing $L$. This indicates that as the NHs getting close, the FM state becomes relatively more stable, i.e., the FM coupling is favored by the super exchange interaction. Note that if the spin coupling is defined only by the bipartite lattice model, the AF state would become more favored when the NHs get closer in the antiparallel configuration, but Fig. 3(c) shows the opposite trend. Also plotted in Fig. 3(c) are magnetic moments on the $\mathrm{NH}$ edges, which are found to increase in the FM but decrease in the AF configuration with decreasing $L$. This again reflects that the edge magnetization on the neighboring $\mathrm{NHs}$ is enhanced with the same spin when they are FM coupled but suppressed with the opposite spin when they are AF coupled by the super exchange

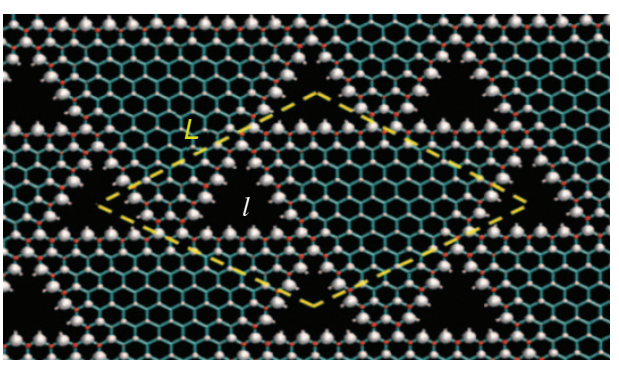

(a)

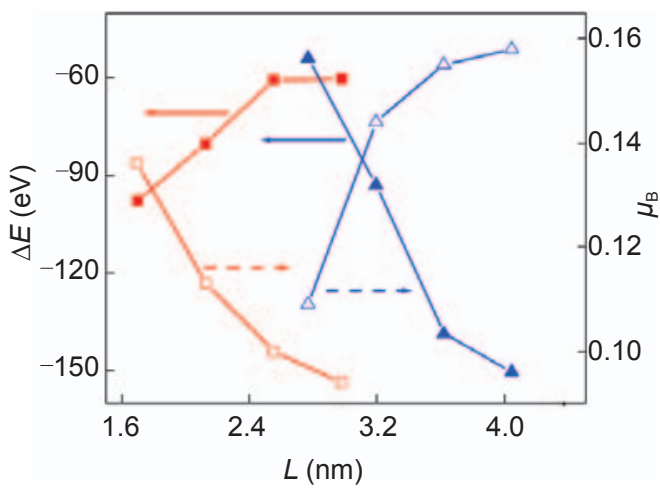

(c)

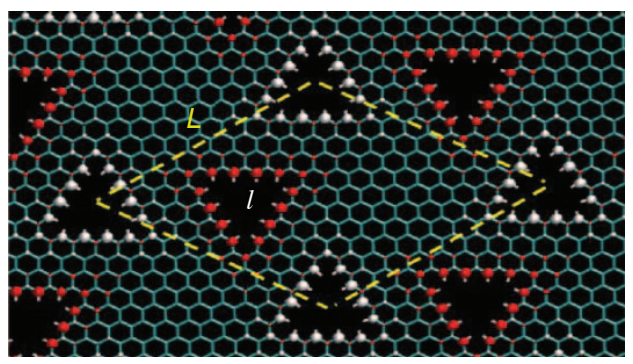

(b)

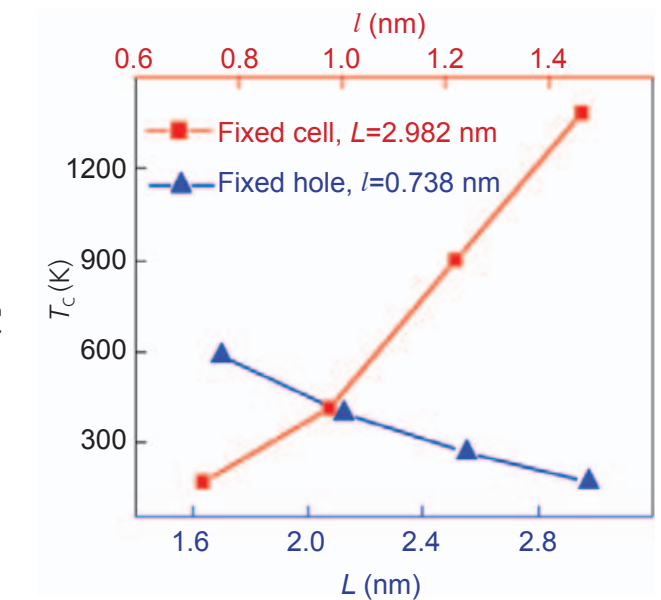

(d)

Figure 3 (a) Ground-state spin configurations in an FM honeycomb NH superlattice. (b) Same as (a) in an AF superlattice. All the symbols and notations for bonds and spin densities are the same as Fig. 1. Yellow dashed lines mark the primitive cell. (c) $\Delta E_{\mathrm{pc}}=E(\mathrm{FM})-E(\mathrm{PM})$ of the FM superlattice (red solid squares) and $\Delta E_{\mathrm{ac}}=E(\mathrm{AF})-$ $E(P M)$ of the AF superlattice (blue solid triangles) vs cell dimension ( $L$ ), edge magnetic moments, $\mu_{B}$, in the FM lattice with fixed hole size $(l=1.476 \mathrm{~nm})$ (red open squares) and in the AF lattice $(l=0.738 \mathrm{~nm})$ (blue open triangles) vs $L$. (d) Curie temperature of the FM superlattice as a function of $\mathrm{NH}$ size $(l)$ and $L$

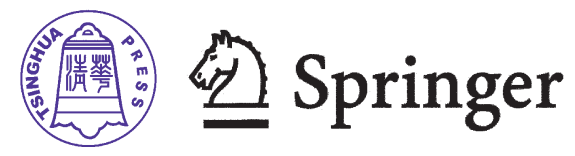


interaction.

The above results show that long-range ferromagnetic ordering can be created by employing the parallel configuration of triangular NHs in different lattice symmetries (see Fig. 2). The next important question is what Curie temperature $\left(T_{C}\right)$ they can have. We have estimated $T_{\mathrm{C}}$ using the mean-field theory of Heisenberg model [21-23],

$$
T_{\mathrm{C}}=\frac{2 \Delta}{3 k_{\mathrm{B}}},
$$

where $\Delta$ is the energy cost to flip one "NH spin" in the FM lattice, which have been calculated directly from first principles for the honeycomb lattices (Fig. $3(\mathrm{a}))$, and $k_{\mathrm{B}}$ is the Boltzman constant. Figure 3(d) shows that $T_{\mathrm{C}}$ increases from $169 \mathrm{~K}$ to $1388 \mathrm{~K}$ when $\mathrm{NH}$ size $(l)$ increases from 0.738 to $1.476 \mathrm{~nm}$ with cell dimension $(L)$ fixed at $2.982 \mathrm{~nm}$, and decreases from $586 \mathrm{~K}$ to $169 \mathrm{~K}$ when $L$ increases from $1.704 \mathrm{~nm}$ to $2.982 \mathrm{~nm}$ with $l$ fixed at $0.738 \mathrm{~nm}$. These trends are expected since magnetization is stronger for larger $\mathrm{NH}$ size and higher $\mathrm{NH}$ density. Limited by computational resource, some of our cell dimensions are possibly unrealistically too small ( $\mathrm{NH}$ density too high), which gives rise to a very high $T_{\mathrm{C}}$. Still, it is possible to make FM GNH superlattices with $T_{\mathrm{C}}$ above room temperature by using an $\mathrm{NH}$ size of $\sim 50$ $\mathrm{nm}$ and a density of $10^{-4} \mathrm{~nm}^{-2}$. We note that a recent experiment [24] has shown a $T_{C} \geqslant 350 \mathrm{~K}$ in $\mathrm{FM}$ graphite made by proton bombardment.

Since graphene-based nanostructures hold great promise for future electronics [25-28], our discovery of GNH magnetism offers the exciting prospect of combining magnetic and semiconducting behavior in one material system. Here, we demonstrate the possibility of making diluted magnetic semiconductors (DMS) by exploiting GNHs with two different kinds of edges. Similar to superlattices of zigzag NHs, we can create superlattices of armchair $\mathrm{NHs}$, which constitute a class of 2-D semiconductors. Figure 4(b) shows the band structure of a superlattice of rhombus armchair NHs (Fig. 4(a)) having a direct band gap of $0.43 \mathrm{eV}$, as obtained from first-principle calculations. Figure 4(c) shows the band gap as a function of NH size $(l)$ and cell dimension $(L)$, from tight-binding calculations [29]. The gap increases with increasing $l$ but decreases with the increasing $L$.
DMS can be made by adding triangular zigzag NHs into the semiconductor superlattice (see Fig. 4(d)). To ensure the ferromagnetism, all triangular NHs must be parallel with each other acting like magnetic dopants. Usually DMS are synthesized by mixing two different materials, typically III$\mathrm{V}$ semiconductors and transition-metal magnetic elements [30,31]. The main challenge is to increase the magnetic dopant concentration in order to raise the Curie temperature, because the two types of materials are usually not miscible. Here, we introduce an "allcarbon" DMS, in which combined semiconductor and magnetic behavior are achieved by structural manipulation. Consequently, room-temperature DMS are reachable because the dopant concentration can be increased without the miscibility problem. One might also consider doping other magnetic elements into the semiconducting GNH superlattice.

It is very exciting to consider making engineered magnetic materials with NHs for various applications. For example, it is possible to directly pattern NHs into engineered magnetic storage media (see supplementary Fig. S-4). The ground state of rhombus NHs is AF (see Fig. 1(b) and lowerleft inset of supplementary Fig. S-4) and their first excited state is FM (up-right inset of supplementary Fig. S-3) when the NH size is larger than 50 atoms (l=14.6 $\AA$ ) according to our calculation. Taking each $\mathrm{NH}$ as one bit, we can assign the ground state with " $S=0$ " and the excited state with " $S=N$ " to represent the " 0 " and " 1 ", respectively. The switching between " 0 " to " 1 " can be done by applying a local magnetic field or energy pulse to convert between the ground and the excited state. Using an NH size of $\sim 50 \mathrm{~nm}$ and a density of $10^{-4} \mathrm{~nm}^{-2}$, a storage density about 0.1 terabit per square inch would be achieved, much higher than the current density in use. However, fabrication of such graphene-based nanostructures will be a challenge, requiring major advances in both graphene synthesis and nanopatterning technologies. We hope our theoretical work will motivate future experimental efforts to overcome the challenge. Another interesting topic of future study is the magnetocrystalline anisotropy around individual $\mathrm{NHs}$; the anisotropy energy must be larger than $k_{\mathrm{B}} T$ for the proposed storage media to work. 


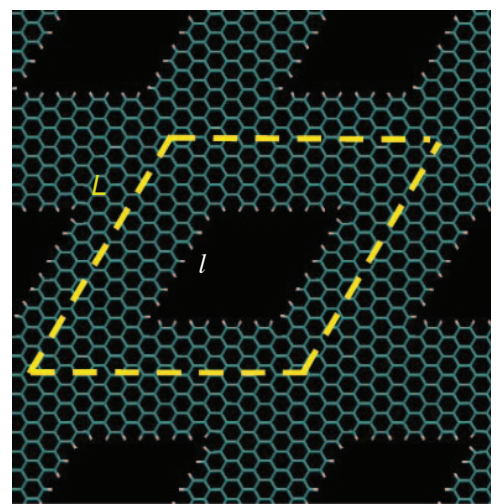

(a)

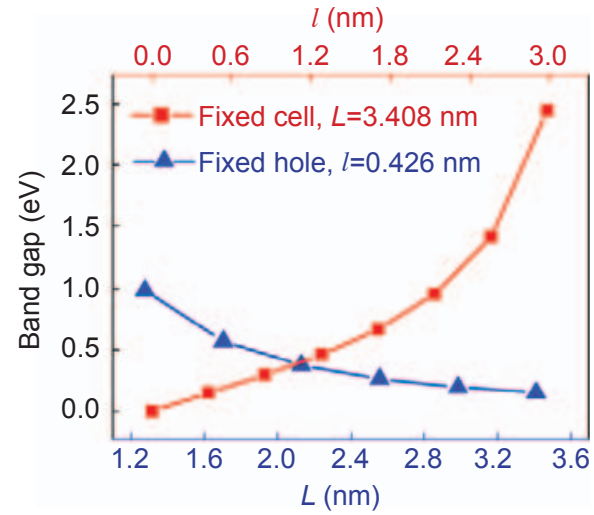

(c)

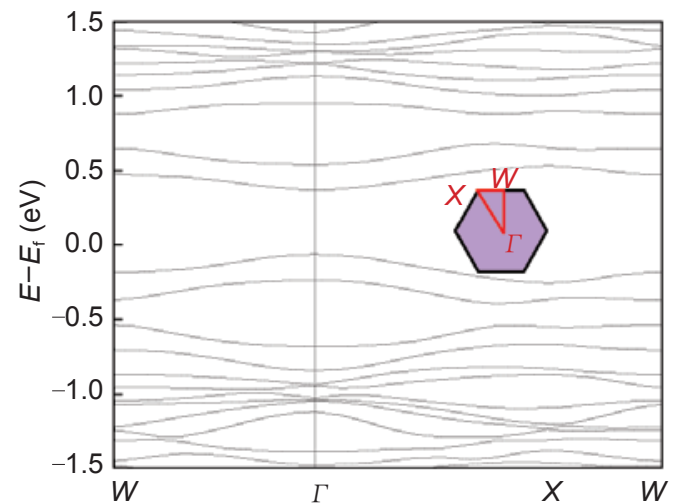

(b)

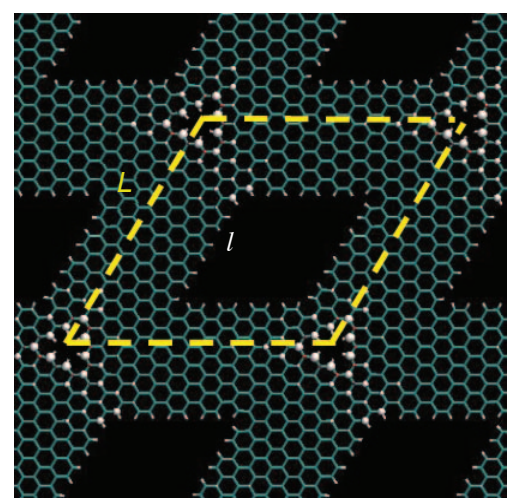

(d)

Figure 4 Illustration of DMS made from GNH superlattice. (a) a semiconductor GNH hexagonal lattice ( $L=8 a, a=2.46 \AA$, is the lattice constant of graphene) consisting of an array of rhombus armchair NHs (l=4a); (b) generalized gradient appoximated (GGA) band structure of (a), the inset shows the Brillouin zone; (c) TB band gap of (a)-type structures as a function of NH size $(l)$ and cell dimension ( $($ ); (d) magnetic semiconductor made by doped (a) with triangular zigzag NHs. All the symbols and notations for bonds and spin densities are the same as Fig. 1

\section{Acknowledgements}

We thank DOE-NERSC and Center for High Performance Computing (CHPC) at the University of Utah for providing the computing resources. This work is supported by DOE.

\section{References}

[1] Slater, J. C. Phys. Rev. 1936, 49, 537-545.

[2] Liu, F.; Khanna, S. N.; Jena, P. Phys. Rev. B 1990, 42, 976 -979 .

[3] Shibayama, Y.; Sato, H.; Enoki, T.; Endo, M. Phys. Rev. Lett. 2000, 84, 1744-1747.

[4] Esquinazi, P.; Spemann, D.; Hohne, R.; Setzer, A.; Han, K. H.; Butz, T. Phys. Rev. Lett. 2003, 91, 227201.

[5] Lehtinen, P. O.; Foster, A. S.; Ma, Y. C.; Krasheninnikov, A.
V.; Nieminen, R. M. Phys. Rev. Lett. 2004, 93, 187202.

[6] Lee, H.; Son, Y. W.; Park, N.; Han, S. W.; Yu, J. J. Phys. Rev. B 2005, 72, 174431.

[7] Coey, J. M. D.; Venkatesan, M.; Fitzgerald, C. B.; Douvalis, A. P.; Sanders, I. S. Nature 2002, 420, 156159.

[8] Kusakabe, K.; Maruyama, M. Phys. Rev. B 2003, 67, 092406.

[9] Son, Y. W.; Cohen, M. L.; Louie, S. G. Nature 2006, 444, 347-349.

[10] Nomura, K.; MacDonald, A. H. Phys. Rev. Lett. 2006, 96, 256602.

[11] Pisani, L.; Chan, J. A.; Montanari, B.; Harrison, N. M. Phys. Rev. B 2007, 75, 064418.

[12] Brey, L.; Fertig, H. A.; Das Sarma, S. Phys. Rev. Lett. 2007, 99, 116802.

\section{国 Springer}


[13] Novoselov, K. S.; Jiang, Z.; Zhang, Y.; Morozov, S. V.; Stormer, H. L.; Zeitler, U.; Maan, J. C.; Boebinger, G. S.; Kim, P.; Geim, A. K. Science 2007, 315, 1379-1379.

[14] Jiang, D. E.; Sumpter, B. G.; Dai, S. J. Chem. Phys. 2007, $127,124703$.

[15] Yazyev, O. V.; Helm, L. Phys. Rev. B 2007, 75, 125408.

[16] Fernandez-Rossier, J.; Palacios, J. J. Phys. Rev. Lett. 2007, 99, 177204.

[17] Wang, W. L.; Meng, S.; Kaxiras, E. Nano Lett. 2008, 8, 241-245.

[18] Huang, B.; Liu, F.; Wu, J.; Gu, B. L.; Duan, W. Phys. Rev. $B$ 2008, 77, 153411.

[19] Kresse, G.; Hafner, J. Phys. Rev. B 1993, 47, 558-561.

[20] Lieb, E. H. Phys. Rev. Lett. 1989, 62, 1201-1204.

[21] Hynninen, T.; Raebiger, H.; von Boehm, J. Phys. Rev. B 2007, 75, 125208

[22] Sato, K.; Dederics, P. H.; Katayama-Yoshida, H. Europhys. Lett. 2003, 61, 403-408.

[23] Turek, I.; Kudrnovsky, J.; Bihlmayer, G.; Blugel, S. J. Phys: Condens. Matter 2003, 15, 2771-2782.

[24] Barzola-Quiquia, J.; Esquinazi, P.; Rothermel, M.;
Spemann, D.; Butz, T.; Garcia, N. Phys. Rev. B 2007, 76, 161403(R).

[25] Novoselov, K. S.; Geim, A. K.; Morozov, S. V.; Jiang, D.; Zhang, Y.; Dubonos, S. V.; Grigorieva, I. V.; Firsov, A. A. Science 2004, 306, 666-669.

[26] Novoselov, K. S.; Geim, A. K.; Morozov, S. V.; Jiang, D.; Katsnelson, M. I.; Grigorieva, I. V.; Dubonos, S. V.; Firsov, A. A. Nature 2005, 438, 197-200.

[27] Ozyilmaz, B.; Jarillo-Herrero, P.; Efetov, D.; Abanin, D. A.; Levitov, L. S.; Kim, P. Phys. Rev. Lett. 2007, 99, 166804.

[28] Yan, Q. M.; Huang, B.; Yu, J.; Zheng, F. W.; Zang, J.; Wu, J.; Gu, B. L.; Liu, F.; Duan, W. H. Nano Lett. 2007, 7, 1469-1473.

[29] Our tight-binding band structure calculations for semiconductor armchair GNH superlattices were performed using the nearest-neighbor-band model with the hopping parameter $=3.0 \mathrm{eV}$.

[30] Jungwirth, T.; Sinova, J.; Masek, J.; Kucera, J.; MacDonald, A. H. Rev. Mod. Phys. 2006, 78, 809-864.

[31] Macdonald, A. H.; Schiffer, P.; Samarth, N. Nat. Mater. 2005, 4, 195-202. 